\title{
Cancer immunotherapy needs to learn how to stick to its guns
}

\author{
Asmi Chakraborty and Charles J. Dimitroff \\ Department of Translational Medicine, Translational Glycobiology Institute at Florida International University, Herbert Wertheim College of Medicine, Florida International University, Miami, Florida, USA
}

\begin{abstract}
Cancer immunotherapy and its budding effectiveness at improving patient outcomes has revitalized our hope to fight cancer in a logical and safe manner. Immunotherapeutic approaches to reengage the immune system have largely focused on reversing immune checkpoint inhibitor pathways, which suppress the antitumor response. Although these approaches have generated much excitement, they still lack absolute success. Interestingly, newly described host-tumor sugar chains (glycosylations) and glycosylationbinding proteins (lectins) play key roles in evading the immune system to determine cancer progression. In this issue of the $J C l$, Nambiar et al. used patient head and neck tumors and a mouse model system to investigate the role of galactose-binding lectin 1 (Gal1) in immunotherapy resistance. The authors demonstrated that Gal1 can affect immune checkpoint inhibitor therapy by increasing immune checkpoint molecules and immunosuppressive signaling in the tumor. Notably, these results suggest that targeting a tumor's glycobiological state will improve treatment efficacy.
\end{abstract}

\section{Tumor microenvironment}

With the approval of ipilimumab for melanoma treatment in 2011, immune checkpoint inhibitor (ICI) therapies have proved beneficial for inducing immune activity against various tumors. ICIs, consisting mostly of neutralizing antibodies against programmed cell death 1 (PD1), programmed death ligand 1 (PD-L1), and CTLA-4, are either administered as monotherapies or in combination with other synergistic therapies (1). However, the complex microenvironment of solid tumors with the abundance of immunosuppressive molecules such as IL-10, IL-6, IL- $1 \alpha$, and IL-1 $\beta$, coupled with a dearth of neoantigens, has limited the success rate of immunotherapies (2). Interestingly, even with the lack of immunoreactive neoantigens, solid tumors actually do express highly aberrant cell-surface glycosylations, a feature now considered a hallmark of neoplas- tic transformation (3). These unique malignancy-specific glycan alterations skew the binding of tumor- and/or host-derived glycan-binding proteins, termed lectins.

Aberrant lectin binding is capable of altering critical cellular functions. For example, expression of sialyl Lewis ${ }^{\mathrm{X}}$ and ${ }^{\mathrm{A}}$ structures enables circulating cancer cells to bind C-type lectins such as endothelial E-selectin, attach to endothelial cells, infiltrate distant sites, and establish metastasis $(4,5)$. Additionally, increased $\alpha 2-6$ sialylation in $\mathrm{O}-$ and $\mathrm{N}$-glycans on cancer cells prevents endogenous immunoregulating lectins, called galectins, from binding (4, 6). Galectins, which bind $\beta$-galactosidebearing glycans, are divided into three subtypes primarily on the basis of their structure (7). There are 15 known galectins reported in mammals and 12 in humans (8). Galectins play an important role in cell-cell and cell-extracellular matrix

- Related Article: p. 5553

Conflict of interest: The authors have declared that no conflict of interest exists. 
A

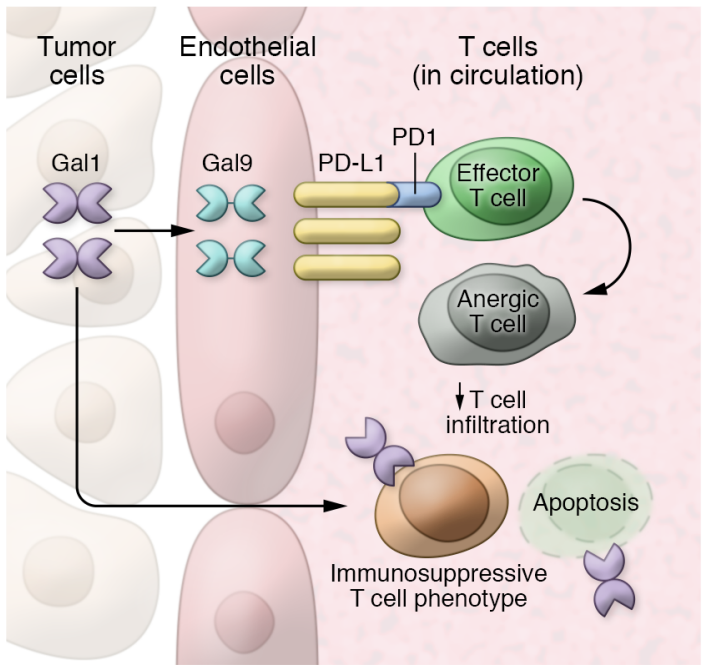

B

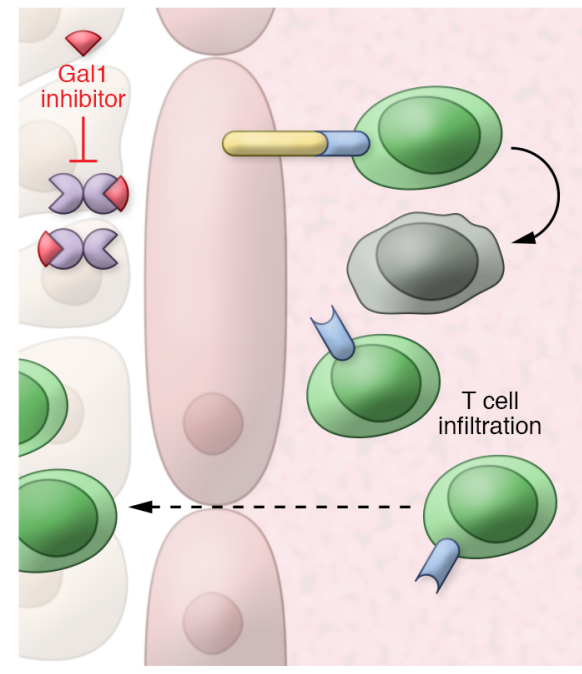

C

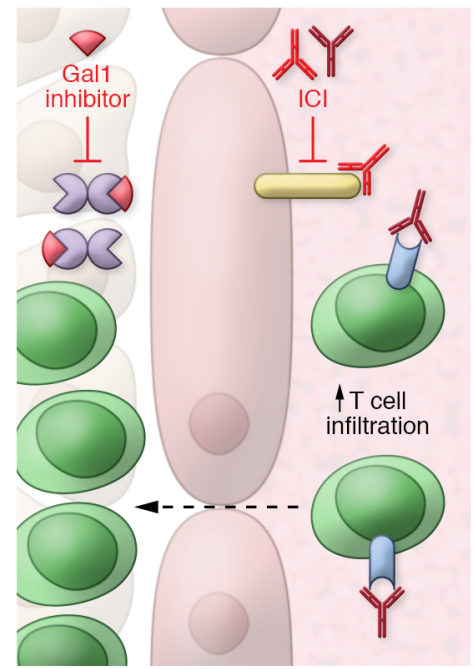

Figure 1. Galectin inhibitors enhance tumor immune surveillance. (A) Gal1 secreted by tumor cells primes the endothelial cells to express PD-L1 and Gal9, both of which lead to T cell anergy as well as reduced T cell infiltration. T cells are also directly targeted by Gal1 to induce an immune-suppressive phenotype as well as apoptosis. (B) Treatment with a Gal1 inhibitor increases T cell infiltration. (C) Treatment with a Gal1 inhibitor in combination with immune checkpoint inhibitors enhances immune surveillance, with increased infiltration of T cells.

The current study by Nambiar et al. demonstrated that head and neck tumor Gal1 promotes $\mathrm{T}$ cell anergy by upregulating PD-L1 and Gal9 expression in tumor endothelial cells (Figure 1A). This is an important finding, as previous studies have neglected endothelial cell signaling as a causative agent in hampering $\mathrm{T}$ cell infiltration. Notably, when Gal1 was blocked, $\mathrm{T}$ cells infiltrated into the tumor microenvironment (Figure 1B) (20). Further, the author's findings are in accordance with tumor-promoting characteristics of Gal9 via Tim-3Gal9 interactions. This result is indeed complex, as other data indicate that increased Gal9 expression is associated with increased patient survival and Gal9 loss with increased metastasis and mortality (21). The protumorigenic function of Gal9, demonstrated by Nambiar and colleagues, is, perhaps, governed by its restricted upregulation in endothelial cells. This aspect of cell-specific galectin expression in tumor progression is intriguing, since Gal9 in exosomes can also establish an immune-privileged microenvironment conducive to metastases formation (22), whereas extracellular Gal9 can induce apoptosis in multiple solid tumor models such as hepatocellular carcinoma, malignant melanoma, and colon cancer, among others (21).

\section{Galectins and their glycan ligands as therapeutic targets}

Altered glycosylation can encourage cancer progression in a number of ways, from promoting chemotherapeutic resistance and enhancing metastatic pathways to the well-described dampening of tumor-immune surveillance (23). It is therefore not surprising that lectins, which bind and help convey malignancy-associated glycan activities, are integral factors in cancer progression and therapy resistance. Gal1 and Gal3 can upregulate multidrug resistance protein 1 , which is a glycoprotein that pumps chemotherapeutics out of cancer cells. Gal3 also interacts with $\mathrm{Na}^{+} / \mathrm{K}^{+}$ATPase and P-glycoprotein function by increasing ATPase activity, thereby reducing chemotherapeutic efficacy (24). Additionally, galectins, especially Gal1, are associated with radiation therapy resistance (25). In the current study by Nambiar et al., reducing tumor-derived Gal1 in a mouse model increased sensitivity to radiation therapy in combination with ICI therapy (20). Targeting Gal1 via genetic ablation or ligand-binding antagonism often results in enhanced immune surveillance (26), which could therefore increase ICI efficacy. Although various clinical trials are underway to test the efficacy of galectin inhibitors in combination with chemotherapy, Gal1 inhibition in concert with ICI treatment has yet to be reported (23). The additional compelling preclinical data reported by Nambiar et al. further strengthen the argument for clinical assessment of Gal1 inhibition in combination with ICI treatment (Figure 1C).

Although targeting galectins is an exciting avenue, there are potential challenges and pitfalls that might arise as a result of the pleiotropic role of galectins associated with cellular localization, tissue of origin, and local microenvironmental concentrations. In addition to the complicated role of Gal9 in tumor progression mentioned above, Gal3, implicated in several solid tumors, also has varying effects, depending on cellular localization and cancer type. Specific cancer models can dictate that extracellular Gal3 is proapoptotic, whereas intracellular Gal3 is antiapoptotic (27). Therefore, anti-galectin therapy must possess specificity for the cancer glycome. Moreover, in certain instances, targeting the glycans that galectins would otherwise bind to might provide better treatment for patients. One way to interfere with the cancer-specific ligand-galectin interaction involves targeting the enzymes (glycosyltransferases) that form glycosidic linkages between individual sugar molecules to build cancer-associated glycans. 
Studies continue to identify various glycosyltransferases and preferred protein scaffolds responsible for cancer cell glycome generation. Blunting glycan-forming activities would curtail key cancer cell-specific glycans and thereby disrupt cancer-promoting glycan-galectin interactions. Notably, sugar analogs such as fluorinated $\mathrm{N}$-acetylglucosmine can antagonize the synthesis of cancer-associated glycans and, by extension, hamper lectin-binding activity (28-30). In conclusion, insights from Nambiar et al. regarding ICI treatment resistance conferred by Gal1 further highlight the need to combine glycobiological pathway-based treatment with ICI therapy. Whether neutralizing the expression or function of Gal1, its glycan ligands, or its downstream immunoregulatory effectors, we can leverage the preclinical data provided by Nambiar et al. to exploit glycan-lectin interactions and optimize cancer immunotherapeutic efficacy.

\section{Acknowledgments}

This Commentary was supported by the National Cancer Institute (NCI)/NIH Alliance of Glycobiologists for Cancer Research: Biological Tumor Glycomics Laboratory (U01 CA225644, to CJ Dimitroff); the National Institute of Allergy and Infectious Diseases (NIAID)/NIH (R21 AI146368, to CJ Dimitroff); and the Mizutani Foundation for Glycoscience Research Grant (to CJ Dimitroff). The content is solely the responsibility of the author and does not necessarily represent the official views of the NIH.

Address correspondence to: Charles J. Dimitroff, Herbert Wertheim College of Medicine, Florida International University, AHC2, 6th Floor, Room 678, 11200 S.W. 8th Street, Miami, Florida 33199, USA. Phone: 305.348.9069; Email: cdimitroff@fiu.edu.
1. Darvin P, Toor SM, Sasidharan Nair V, Elkord E. Immune checkpoint inhibitors: recent progress and potential biomarkers. Exp Mol Med. 2018;50(12):165.

2. Vinay DS, et al. Immune evasion in cancer: mechanistic basis and therapeutic strategies. Semin Cancer Biol. 2015;35 Suppl:S185-S198.

3. Hakomori S. Aberrant glycosylation in tumors and tumor-associated carbohydrate antigens. Adv Cancer Res. 1989;52:257-331.

4. Dimitroff CJ. Galectin-binding O-glycosylations as regulators of malignancy. Cancer Res. 2015;75(16):3195-3202.

5. Barthel SR, et al. Definition of molecular determinants of prostate cancer cell bone extravasation. Cancer Res. 2013;73(2):942-952.

6 . Bellis SL. Variant glycosylation: an underappreciated regulatory mechanism for beta1 integrins. Biochim Biophys Acta. 2004;1663(1-2):52-60.

7. Johannes L, Jacob R, Leffler H. Galectins at a glance. J Cell Sci. 2018;131(9):jcs208884.

8. Cummings RD, et al. In Varki A, et al, eds. Essentials of Glycobiology. La Jolla, CA: Cold Spring Harbor Laboratory Press; 2009.

9. Arthur CM, Baruffi MD, Cummings RD, Stowell SR. Evolving mechanistic insights into galectin functions. In: Stowell SR, Cummings RD, eds. Galectins Methods in Molecular Biology (Methods and Protocols). New York, NY;Humana Press;2015:1-35.

10. Ebrahim AH, et al. Galectins in cancer: carcinogenesis, diagnosis and therapy. Ann Transl Med. 2014;2(9):88.

11. Chou FC, Chen HY, Kuo CC, Sytwu HK. Role of galectins in tumors and in clinical immunotherapy. Int J Mol Sci. 2018;19(2):E430.

12. Sweeney JG, et al. Loss of GCNT2/I-branched glycans enhances melanoma growth and survival. Nat Commun . 2018;9(1):3368.

13. Cedeno-Laurent F, Dimitroff CJ. Galectins and their ligands: negative regulators of anti-tumor immunity. Glycoconj J. 2012;29(8-9):619-625.

14. Chakraborty A, et al. ST6Gal-I sialyltransferase promotes chemoresistance in pancreatic ductal adenocarcinoma by abrogating gemcitabine-mediated DNA damage. J Biol Chem. 2018;293(3):984-994.

15. Schultz MJ, et al. The tumor-associated glycosyltransferase ST6Gal-I regulates stem cell transcription factors and confers a cancer stem cell phenotype. Cancer Res. 2016;76(13):3978-3988.

16. Viguier M, Advedissian T, Delacour D, Poirier F, Deshayes F. Galectins in epithelial functions. Tissue Barriers. 2014;2:e29103.
17. Laderach DJ, et al. Dissecting the signal transduction pathways triggered by galectin-glycan interactions in physiological and pathological settings. IUBMB Life. 2010;62(1):1-13.

18. Cedeno-Laurent F, Dimitroff CJ. Galectin-1 research in $\mathrm{T}$ cell immunity: past, present and future. Clin Immunol. 2012;142(2):107-116.

19. Cedeno-Laurent F, Watanabe R, Teague JE, Kupper TS, Clark RA, Dimitroff CJ. Galectin-1 inhibits the viability, proliferation, and Th1 cytokine production of nonmalignant $\mathrm{T}$ cells in patients with leukemic cutaneous T-cell lymphoma. Blood. 2012;119(15):3534-3538.

20. Nambiar DK, et al. Galectin-1-driven T cell exclusion in the tumor endothelium promotes immunotherapy resistance. JClin Invest. 2019;129(12):5553-5567.

21. Zhou X, et al. Galectin-9 expression predicts favorable clinical outcome in solid tumors: a systematic review and meta-analysis. Front Physiol. 2018;9:452.

22. Barros FM, Carneiro F, Machado JC, Melo SA Exosomes and immune response in cancer: friends or foes? Front Immunol. 2018;9:730.

23. Mereiter S, Balmaña M, Campos D, Gomes J, Reis CA. Glycosylation in the era of cancer-targeted therapy: where are we heading? Cancer Cell. 2019;36(1):6-16.

24. Wdowiak K, et al. Galectin targeted therapy in oncology: current knowledge and perspectives. Int J Mol Sci. 2018;19(1):E210.

25. Chou SY, Yen SL, Huang CC, Huang EY. Galectin-1 is a poor prognostic factor in patients with glioblastoma multiforme after radiotherapy. BMC Cancer. 2018;18(1):105.

26. Cedeno-Laurent F, et al. Metabolic inhibition of galectin-1-binding carbohydrates accentuates antitumor immunity. J Invest Dermatol. 2012;132(2):410-420.

27. Nakahara S, Oka N, Raz A. On the role of galectin-3 in cancer apoptosis. Apoptosis. 2005;10(2):267-275.

28. Barthel SR, et al. Peracetylated 4-fluoroglucosamine reduces the content and repertoire of $\mathrm{N}$ - and O-glycans without direct incorporation. J Biol Chem. 2011;286(24):21717-21731.

29. Descheny L, Gainers ME, Walcheck B, Dimitroff CJ. Ameliorating skin-homing receptors on malignant $\mathrm{T}$ cells with a fluorosugar analog of $\mathrm{N}$-acetylglucosamine: $\mathrm{P}$-selectin ligand is a more sensitive target than E-selectin ligand. J Invest Dermatol. 2006;126(9):2065-2073.

30. Dimitroff CJ. Leveraging fluorinated glucosamine action to boost antitumor immunity. Curr Opin Immunol. 2013;25(2):206-213. 